TITLE:

\title{
Human and mouse induced pluripotent stem cells are differentially reprogrammed in response to kinase inhibitors.
}

\section{AUTHOR(S):}

Hirano, Kunio; Nagata, Shogo; Yamaguchi, Shinpei; Nakagawa, Masato; Okita, Keisuke; Kotera, Hidetoshi; Ainscough, Justin; Tada, Takashi

\section{CITATION:}

Hirano, Kunio ... [et al]. Human and mouse induced pluripotent stem cells are differentially reprogrammed in response to kinase inhibitors.. Stem cells and development 2012, 21(8): 1287-1298

ISSUE DATE:

2012-05-20

URL:

http://hdl.handle.net/2433/157679

\section{RIGHT:}

This is a copy of an article published in 'Stem cells and development'; (c) 2012 Mary Ann Liebert, Inc.; 'Stem cells and development' is available online at: http://online.liebertpub.com. 


\title{
Human and Mouse Induced Pluripotent Stem Cells Are Differentially Reprogrammed in Response to Kinase Inhibitors
}

\author{
Kunio Hirano, ${ }^{1, *}$ Shogo Nagata, ${ }^{1,2, *}$ Shinpei Yamaguchi, ${ }^{1,2}$ Masato Nakagawa, ${ }^{3}$ Keisuke Okita, ${ }^{3}$ \\ Hidetoshi Kotera, ${ }^{2,4}$ Justin Ainscough, ${ }^{5}$ and Takashi Tada ${ }^{1,2}$
}

Conventional human induced pluripotent stem cells (hiPSCs), reprogrammed from somatic cells by induced expression of Oct4, Sox2, Klf4, and c-Myc, are phenotypically different from mouse embryonic stem cells (ESCs). In mice, culture in N2B27 serum-free 2i media (mitogen-activated protein kinase/extracellular signal-regulated kinase and glycogen synthase kinase 3 inhibitors; PD0325901 and CHIR99021) plus leukemia inhibitory factor (LIF) (2i + LIF medium) enriches for germline competent ESCs. Here, we demonstrate that flat-shaped hiPSC colonies can be reprogrammed into bowl-shaped multi-potent stem cells (2i-hiPSCs) by using $2 \mathrm{i}+\mathrm{LIF}$ medium. Mechanical dissociation of 2i-hiPSC colonies enables stable maintenance for $>20$ passages. Importantly, gene expression profiling demonstrated that 2i-hiPSCs more closely resemble primitive neural stem cells (PNSCs). Notably, this 2i-induced phenotype was generated from conventional hiPSCs, but not human ESCs (hESCs), thus correlating with the observation of neuroectodermal SOX1-positive colonies in conventional hiPSCs, but not hESCs in $2 \mathrm{i}+$ LIF medium. Thus, 2i-hiPSCs, which are nonteratoma forming PNSCs, may represent a safe source of cells for neural research and regenerative medicine.

\section{Introduction}

G ROUND STATE MOUSE embryonic stem cells (GS-mESCs) are pluripotent cell populations with homogeneous stem cell identity, including germline competency. They are able to proliferate with minimal extrinsic stimuli when cultured in serum-free neurobasal N2B27 medium in the presence of 2 small-molecule inhibitors (2i) against mitogen-activated protein kinase/extracellular signal-regulated kinase (ERK) kinase (MEK) and glycogen synthase kinase 3 (GSK3), plus leukemia inhibitory factor (LIF) ( $2 \mathrm{i}+\mathrm{LIF}$ medium) [1]. GSmESCs have been derived from not only the inbred 129-strain, but also the nonpermissive strains, including nonobese diabetic mice [2]. Further, germline-competent ESC lines have been successfully generated from rat blastocysts by using similar $2 i+$ LIF medium $[3,4]$. Therefore, it is likely that pluripotency, as seen in ESCs, is a state that utilizes common molecular mechanisms which are shared between rodents. If the pluripotent state maintained under $2 \mathrm{i}+\mathrm{LIF}$ conditions is not only a rodent-specific phenomenon, then it is theoretically possible to generate pluripotent stem cells (PSCs) that re- semble GS-mESCs from human ESCs (hESCs), as well as from human induced PSCs (hiPSCs) derived from reprogrammed somatic cells.

Oct4, Sox2, Klf4, and c-Myc (OSKM)-hiPSCs resemble blastocyst-derived hESCs in colony morphology, global gene expression and capacity for teratoma formation [5,6]. Intriguingly, hESC and hiPSC colonies exhibit characteristic flatshaped morphology, which is clearly distinct from mESC and mouse iPSC (miPSC) colonies that have bowl-shaped morphology [7]. Further, hiPSCs and hESCs can be maintained in human ES medium with knockout serum replacement (KSR) and basic fibroblast growth factor (bFGF) but not in mESC medium with fetal bovine serum (FBS) and LIF. Thus, hESCs and hiPSCs differ markedly from mESCs and miPSCs with regard to cellular physiology. Consistent with this, several lines of evidence have suggested that hESCs are more closely related to mouse epiblast-stem cells (mEpiSCs) than they are to mESCs [8-11]. Notably, mESC-like naïve hESCs have been generated from conventional hESC s by growth in $2 i+$ LIF medium in the presence of Forskolin, and from conventional hiPSCs by Doxinducible expression of OSKM in $2 \mathrm{i}+$ LIF medium, although

\footnotetext{
${ }^{1}$ Laboratory of Stem Cell Engineering, Institute for Frontier Medical Sciences, Kyoto University, Kyoto, Japan

${ }^{2}$ JST CREST, Saitama, Japan.

${ }^{3}$ Reprogramming Science, Center for iPS Cell Research and Application, Kyoto University, Kyoto, Japan.

${ }^{4}$ Micro Engineering, Faculty of Engineering, Kyoto University, Kyoto, Japan.

${ }^{5}$ Cardiovascular and Neuronal Remodelling, LIGHT, Leeds University, Leeds, United Kingdom.

*These two authors contributed equally to this work.
} 
establishment of stable lines from these naïve cells has been unsuccessful to date [12]. Similarly, mESC-like hESCs can be generated from conventional hESCs in Dulbecco's modified Eagle's medium (DMEM) with KSR, LIF, MEK inhibitor PD0325901 and p38 inhibitor SB203580, although the properties of these cells have yet to be fully characterized [13]. Thus, it remains unclear whether mESC-like hiPSCs can be reproducibly generated, and what characteristics mESC-like hiPSCs established in $2 i+$ LIF medium may have.

Here, we demonstrated that bowl-shaped hiPSCs (2ihiPSCs) can be reproducibly isolated from conventional and integration-free (Infr) hiPSCs, but not from hESCs, in $2 \mathrm{i}+\mathrm{LIF}$ medium on feeder-free matrigel-coated dishes. Stable maintenance of 2i-hiPSC lines was enabled by mechanical but not enzymatic dissociation for $>50$ passages over a period of 9 months. High-level PAX6 and SOX2 expression and lowlevel OCT4 and NANOG expression demonstrated that $2 \mathrm{i}-$ hiPSCs had characteristics closer to multi-potent primitive neural stem cells (PNSCs) [14] also known as neural precursors [15] than miPSCs and mESCs. On withdrawal of $2 i+$ LIF, 2i-hiPSCs efficiently differentiated into neurons and astrocytes in vitro. Further, 2i-hiPSCs were found to be incapable of forming teratomas in vivo, when implanted under the kidney capsule of immunodeficient mice. Notably, the differential ability of hiPSC and hESC lines to form PNSCs in $2 i+$ LIF medium is due to differential induction of Sox1positive ecotodermal cells.

\section{Materials and Methods \\ Cell lines}

miPSC lines were established from amniotic cells, which had been obtained from E18.5 embryos by mating of Oct4-GFP (C57BL/6) and Rosa26 (129/Sv) transgenic mice, by retroviral transduction of mouse OSKM and DsRed [16]. R1 mESC $(129 / \mathrm{Sv})$ and miPSC lines were maintained in mES medium [DMEM/F12 supplemented with 15\% FBS, L-glutamine, penicillin-streptomycin, sodium bicarbonate, sodium pyruvate, 2-mercaptoethanol, and 1,000 U/mL of LIF (Chemicon)]. mEpiSC lines were derived from epiblasts of E6.5 embryos obtained from mating of DBA and C57BL/ 6 with the ubiquitously expressing GFP transgene and cultured in mEpi medium [hES medium supplemented with $8 \mathrm{ng} / \mathrm{mL}$ Activin A (Peprotech)] on mouse embryonic fibroblast (MEF) feeder cells $[8,9]$. Human fetal lung fibroblasts (TIG1 and TIG3) provided by the JCRB Cell Bank were used to derive hiPSC lines through retroviral induction of OSKM or OSK [16]. Infr hiPSC lines were established by OSKM transduction with episomal vectors from female adult dermal fibroblasts, HDF1388 [17]. hESC lines, KhES1, and KhES3 [18] were used according to the guidelines for derivation and utilization of hESCs from the ministry of education, culture, sports, science, and technology of Japan. hESC and hiPSC lines were stably maintained in MEF-conditioned hES medium [DMEM/F12 supplemented with 20\% KSR (Invitrogen), L-glutamine, nonessential amino acids, 2-mercaptoethanol, and $10 \mathrm{ng} / \mathrm{mL}$ bFGF (Peprotech)] on matrigel-coated dishes. To address the effect of apoptosis on survival, $10 \mu \mathrm{M}$ ROCK inhibitor Y27632 (Wako) and $10 \mu \mathrm{M}$ Myosin IIA inhibitor Blebbistatin (Toronto Research Chemicals) were added to $2 i+$ LIF medium. All experiments were performed with PSCs at 10-25 passages.

\section{Cell culture in $2 i+$ LIF medium}

N2B27 medium was prepared as a 1:1 mixture of N2 medium [DMEM/F12 medium with N2 supplement (Invitrogen)] and B27 medium [Neurobasal medium with B27 supplement (Invitrogen)] supplemented with L-glutamine and penicillinstreptomycin. $2 \mathrm{i}+$ LIF medium was prepared by supplementing N2B27 medium with $1 \mu \mathrm{M}$ MEK inhibitor PD0325901 (Cayman chemical), $3 \mu \mathrm{M}$ GSK3 inhibitor CHIR99021 (Axon biochemicals), and 1,000 U/mL LIF (Chemicon).

For isolating GS-miPSC and GS-mESC lines, mES culture medium was replaced with $2 \mathrm{i}+\mathrm{LIF}$ medium. For establishing GS-mEpiSC lines, $2.0 \times 10^{5}$ mEpiSCs were plated on $3.5 \mathrm{~cm}$ culture dishes containing feeder cells, cultured in mEpi medium overnight, and then cultured in $2 i+$ LIF medium. Cells were split and reseeded into four $3.5 \mathrm{~cm}$ dishes with feeder cells after 3 days, at which time they were cultured in $2 i+L I F$ medium. GS-mEpiSC colonies were picked 7 days after reseeding and expanded in $2 \mathrm{i}+$ LIF medium. Established GSmEpiSC lines were sub-cultured with $2 i+$ LIF medium every 3 days.

For establishing 2i-hiPSC lines, $2.0 \times 10^{5}$ conventional hiPSCs were plated on matrigel-coated $3.5 \mathrm{~cm}$ dishes in $2 \mathrm{i}+\mathrm{LIF}$ medium. Candidate colonies were picked 5 days after $2 i+$ LIF culture by using a micropipette. Colonies were mechanically dissociated into $40-50$ cells by repeated micropipetting. This process was repeated until 2i-hiPSC lines were established. Established lines were subsequently subcultured by mechanical dissociation in $2 \mathrm{i}+$ LIF medium every 5-7 days.

\section{Chimera and teratoma formation}

For producing chimeric embryos, GS-miPSCs and GSmEpiSCs ubiquitously expressing $\beta$-geo and EGFP, respectively, were microinjected into C57BL/6J x BDF1 blastocysts. Embryos were collected at E15.5 and analyzed by X-gal staining and GFP fluorescence [19].

For obtaining teratomas, undifferentiated 2i-hiPSC lines (about $5.0 \times 10^{6}$ cells / site) and derived EBs $\left(1.0 \times 10^{7}\right.$ cells/ site) were transplanted under the kidney capsule of immunodeficient SCID mice (Clea) by a transfer pipette. Teratoma formation was assessed after 8-10 weeks.

\section{Gene expression}

For reverse transcription (RT)-polymerase chain reaction (PCR) analyses, total RNA of cultured cells was extracted with TRIzol reagent (Invitrogen). cDNA was synthesized from 500 ng total RNA with Superscript III (Invitrogen) by using random hexamers while following the manufacturer's instructions. For mEpiSC RNA extraction, mEpiSC colonies grown on feeder cells were purified by mechanical picking and pooled in TRIzol. Primer sequences used in this study are summarized in Supplementary Table S1; Supplementary Data are available online at www.liebertonline.com/scd.

For global gene expression analyses, $250 \mathrm{ng}$ total RNA was labeled according to standard Affymetrix protocols and hybridized to the Affymetrix mouse genome 4302.0 Array (samples of mouse origin) or the human genome U133 Plus 2.0 Array (samples of human origin). Raw data were normalized by the MAS 5.0 method by using bioconductor package on $\mathrm{R}$ program (www.r-project.org/). Heat maps of 
gene expression profile for all genes in each cell line were visualized by $\mathrm{MeV}$ program (www.tm4.org/mev/). Hierarchical clusters were calculated by Pearson's correlation coefficient ( $\mathrm{r}$ ) and visualized by a pvclust package on $\mathrm{R}$.

\section{Immunocytochemistry}

Cultured cells were fixed with $4 \%$ paraformaldehyde (PFA)/phosphate-buffered saline (PBS) for $10 \mathrm{~min}$ at room temperature, washed with PBST (0.1\% Triton X-100 in PBS), and then pretreated with blocking solution $[3 \%$ bovine serum albumin and $2 \%$ skim milk (DIFCO) in PBST] at $4^{\circ} \mathrm{C}$ overnight. The cells were then stained with fluorescenceconjugated secondary antibodies (1:500; Invitrogen), following an immunoreaction with primary antibodies; anti-OCT4 (1:50; Santa Cruz Biotechnology), anti-SOX2 (1:500; Abcam), anti-Klf4 (1:200; Santa Cruz Biotechnology), anti-NANOG (1:200; ReproCELL), anti-SSEA1 (1:200; DSHB), anti-SSEA4 (1:200; DSHB), anti-E-CADHERIN (1:50; Takara), anti-TRA160 (1:500; Millipore), anti-Tubulin J1 (TuJ1) (1:600; COVANCE), anti-neurofilament-M (NF-M) (1:1,000; Millipore), anti-glial fibrillary acidic protein (GFAP) (1:1,000; Dako), anti-O4 (1:60; Millipore), anti-H3K27me3 (1:200; Millipore), anti-SOX1 (1:100; R\&D systems), and anti-N-CADHERIN (1:100; Chemicon). The cells were counterstained with 4,6diamidino-2-phenylindole and mounted with SlowFade light antifade kit (Invitrogen).

\section{XIST RNA FISH}

Cells were fixed with $4 \%$ PFA/PBS. RNA FISH was performed according to standard procedures with an RNA probe prepared by in vitro transcription with Cy3-UTP (GE Healthcare) and a human full-length XIST cDNA construct [20] using T7 RNA polymerase.

\section{Cell differentiation in vitro}

For neural differentiation, 2i-hiPSCs were cultured on matrigel-coated dishes in N2B27 medium for 4 days after withdrawal of $2 i+$ LIF. Differentiated cells were fixed with $4 \%$ PFA/PBS and analyzed by immunocytochemistry.

For EB formation, small 2i-hiPSC aggregates were formed by overnight hanging drop cultures in DMEM containing $10 \%$ FBS. Aggregates were grown and differentiated in bacterial culture dishes for 5-7 days.

\section{Results}

\section{Mouse GS pluripotent stem cells in 2i+ LIF medium}

To investigate the suitability of serum-free neurobasal N2B27 medium with $2 i+$ LIF (2i+LIF medium) for generation of GS PSCs, we tested 3 types of mouse conventional PSCs: mESCs, miPSCs, and mEpiSCs. GS-mESCs, GSmiPSCs, and GS-mEpiSCs, similarly characterized by bowlshaped morphology of colonies expressing Oct4, Sox2, and Nanog proteins, were reproducibly isolated (Fig. 1A). GSmEpiSCs were isolated as a small number of colonies from conventional mEpiSCs as a consequence of stringent selection in 2i+LIF medium, whereas only mild cell selection was required for the transition from conventional mESCs and miPSCs to GS-mESCs and GS-miPSCs, respectively. These results were verified by triplicate experiments. Before being cultured in $2 \mathrm{i}+\mathrm{LIF}$ medium, miPSCs exhibited incomplete silencing of exogenous Oct4, Klf4, and c-Myc, whereas mEpiSCs exhibited low expression of Rex1, NrOb1, and Stella accompanied by high expression of Lefty1, Sox17, and Fgf5. After culture in 2i+LIF medium, both GS-miPSCs and GSmEpiSCs exhibited similar gene expression patterns to GSmESCs (Fig. 1B). This was verified by analyses of global gene expression profile with expression of microarray assays (Fig. 1C). The efficient contribution to a broad range of cell types in E15.5 chimeric embryos demonstrated pluripotency; of GS-miPSCs with the LacZ reporter gene and GS-mEpiSCs with the GFP reporter gene (Fig. 1D). Our observations on GS-mESCs and mEpiSCs are consistent with findings previously reported $[1,8,9,21,22]$, thus clearly indicating that the $2 \mathrm{i}+\mathrm{LIF}$ medium used here is suitable for generating and maintaining mouse GS PSCs.

\section{Generation of human iPSC lines in 2i+ LIF medium}

To determine whether human somatic cells can be directly reprogrammed into bowl-shaped 2i-hiPSCs, the human primary fetal lung fibroblast lines, female TIG1 and male TIG3, were cultured for 20 days after retroviral transduction of OSKM in human ES medium with KSR and bFGF. Twentyfour hours after replacing the culture medium with $2 i+L I F$, surviving colonies were picked and expanded by gentle dissociation with a glass pipette for 3-4 passages. This resulted in numerous 2i-hiPSC colonies with bowl-shaped mESC-like morphology (Fig. 2A).

To further investigate whether these 2i-hiPSCs had been directly derived from the conventional flat-shaped OSKMhiPSCs established from the TIG1 and TIG3 cells, the culture medium of conventional hiPSCs was switched from human ES medium with KSR and bFGF to $2 \mathrm{i}+$ LIF medium. Conventional hiPSC colonies were severely selected for 5 days of culture. However, after the longer selection period (5-7 days), a small number of bowl-shaped colonies (5-10 colonies from about $2.0 \times 10^{5}$ conventional hiPSCs) were observed. The bowl-shaped colonies were picked and expanded in fresh $2 \mathrm{i}+$ LIF medium after mechanical dissociation by using a glass micropipette (Fig. 2A). Bowl-shaped 2i-hiPSC colonies were reproducibly established from OSKM-hiPSC lines derived from both independent somatic cell lines, TIG1 and TIG3. In total, more than fifty 2i-hiPSC lines were established. Of 5 2i-hiPSC lines examined, all were karyotypically normal $2 n=46, \mathrm{XY}$ or XX (data not shown).

To examine whether 2i-hiPSCs can be generated from iPSC lines that do not contain the integrated exogenous $c$ Myc gene, conventional iPSCs produced by retroviral OSK transduction were cultured in $2 \mathrm{i}+$ LIF medium. Notably, 2ihiPSC lines were reproducibly isolated from conventional OSK-hiPSCs at a similar frequency to that observed from conventional OSKM-hiPSCs. The 2i-hiPSC colonies resembled those derived from OSKM-hiPSCs, with characteristic bowl-shaped colony morphology and cell growth (Fig. 2A). Thus, integration of $c-M y c$ is dispensable for 2i-hiPSC generation from conventional hiPSCs.

To expand the number of 2i-hiPSCs, we also attempted enzymatic dissociation with trypsin and collagenase. Strikingly, none of the cells were capable of complete re-attachment to matrigel-coated culture dishes. Subculture in the presence 


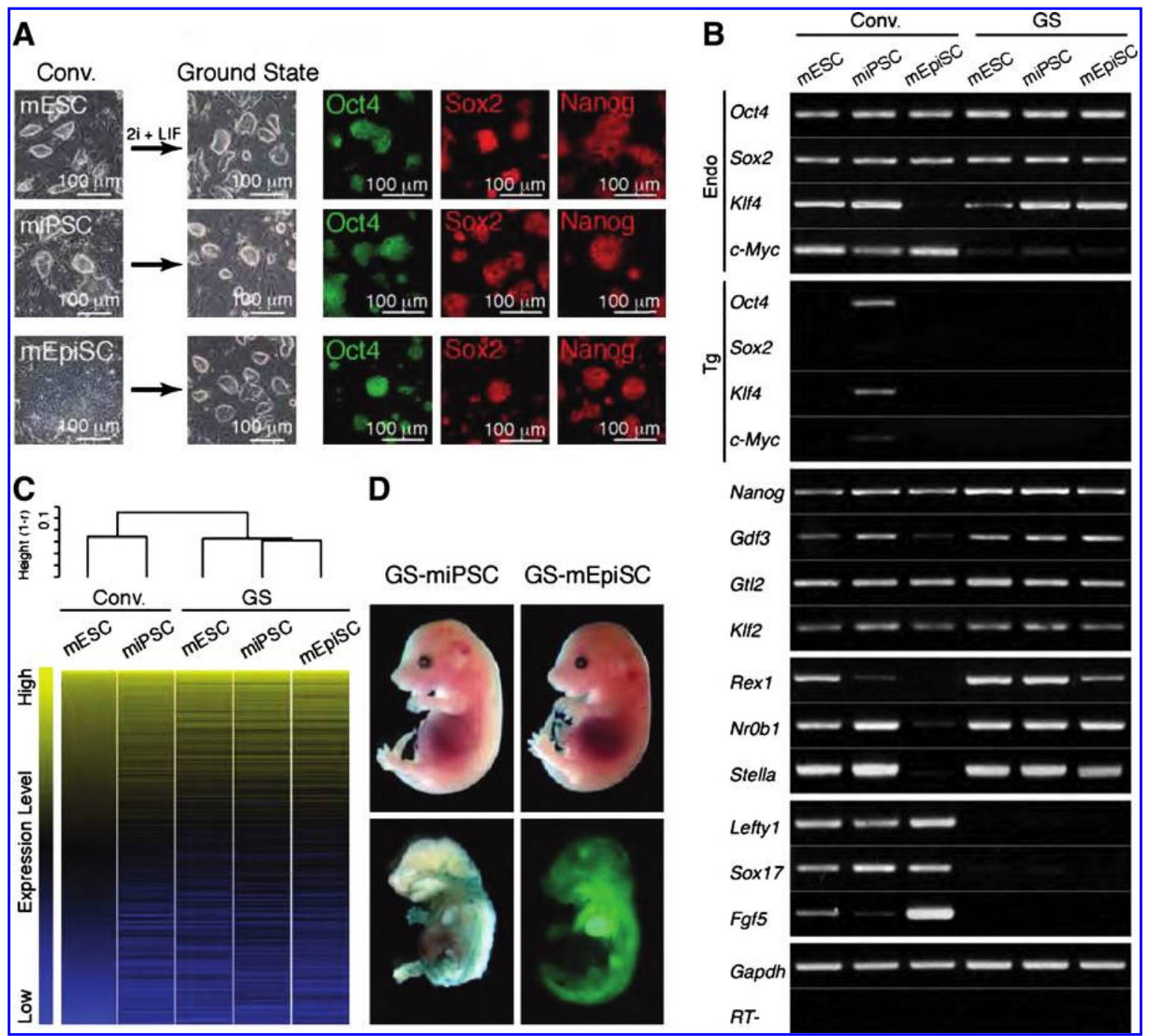

FIG. 1. Generation of mouse ground state (GS) PSC lines. (A) Generation of mouse GS PSCs from conventional mESCs, miPSCs, and mEpiSCs in 2i+LIF medium (GS medium). Bowl-shaped colonies of GS-mESCs, GS-miPSCs and GS-mEpiSC colonies are positive for pluripotent marker proteins, Oct4, Sox2, and Nanog. (B) Gene expression analysis in mESC, miPSC, and mEpiSC cultured with conventional (Conv.) and GS medium. (C) Comparative analysis of global gene expression profile of mESC, miPSC, and mEpiSC cultured with conventional and GS medium by gene expression microarray assay. (D) E15.5 chimeric embryos generated by microinjection of GS-miPSCs and GS-mEpiSCs into host blastocysts. GS-miPSC-derivatives are detected as blue cells by X-gal staining, whereas GS-mEpiSC-derivatives as green fluorescent cells. miPSC, mouse induced pluripotent stem cell; LIF, leukemia inhibitory factor; mESC, mouse embryonic stem cell. mEpiSC, mouse epiblast-stem cell.

of the small molecule Tzv [13] was found to improve attachment, but not survival. In fact, Tzv induced rapid cell differentiation toward neuronal lineages (data not shown). This suggested that subculture by enzymatic dissociation is not suitable for expansion and long-term maintenance of 2i-hiPSC lines. Consequently, 2i-hiPSC colonies were routinely dissociated to clusters of $20-50$ cells by using a glass micropipette under a stereomicroscope. For stable maintenance, dissociated $2 \mathrm{i}$-hiPSC clusters were sub-cultured into fresh $2 i+$ LIF medium in matrigel-coated dishes every 5-7 days. In this manner, we were able to stably maintain and expand 2i-hiPSC clones for $>50$ passages over a period of 9 months under feeder-free conditions. Further, 2i-hiPSCs were successfully cryo-preserved and thawed with hESC freezing medium.

\section{Conventional hESCs and nonintegrated hiPSCs in $2 i+$ LIF medium}

To determine whether 2i-hESCs can be generated from conventional hESCs without integration of exogenous genes, conventional hESCs were cultured in $2 \mathrm{i}+\mathrm{LIF}$ medium. No $2 \mathrm{i}-$ hESC colonies were detected despite 3 repeated experiments with 2 different hESC lines, KhES1 and KhES3 [18], with or without ROCK/Myosin IIA inhibitors [23,24]; whereas 2ihiPSCs were repeatedly generated in the same $2 i+$ LIF medium (Fig. 2B). Further, no 2i-hESC colonies appeared even after addition of Forskolin to the $2 i+$ LIF medium [12]. These findings could be explained by 2 possibilities: (1) transient expression of exogenous OSK is required for 2i-hiPSC generation, or (2) generation potential into multipotent stem 


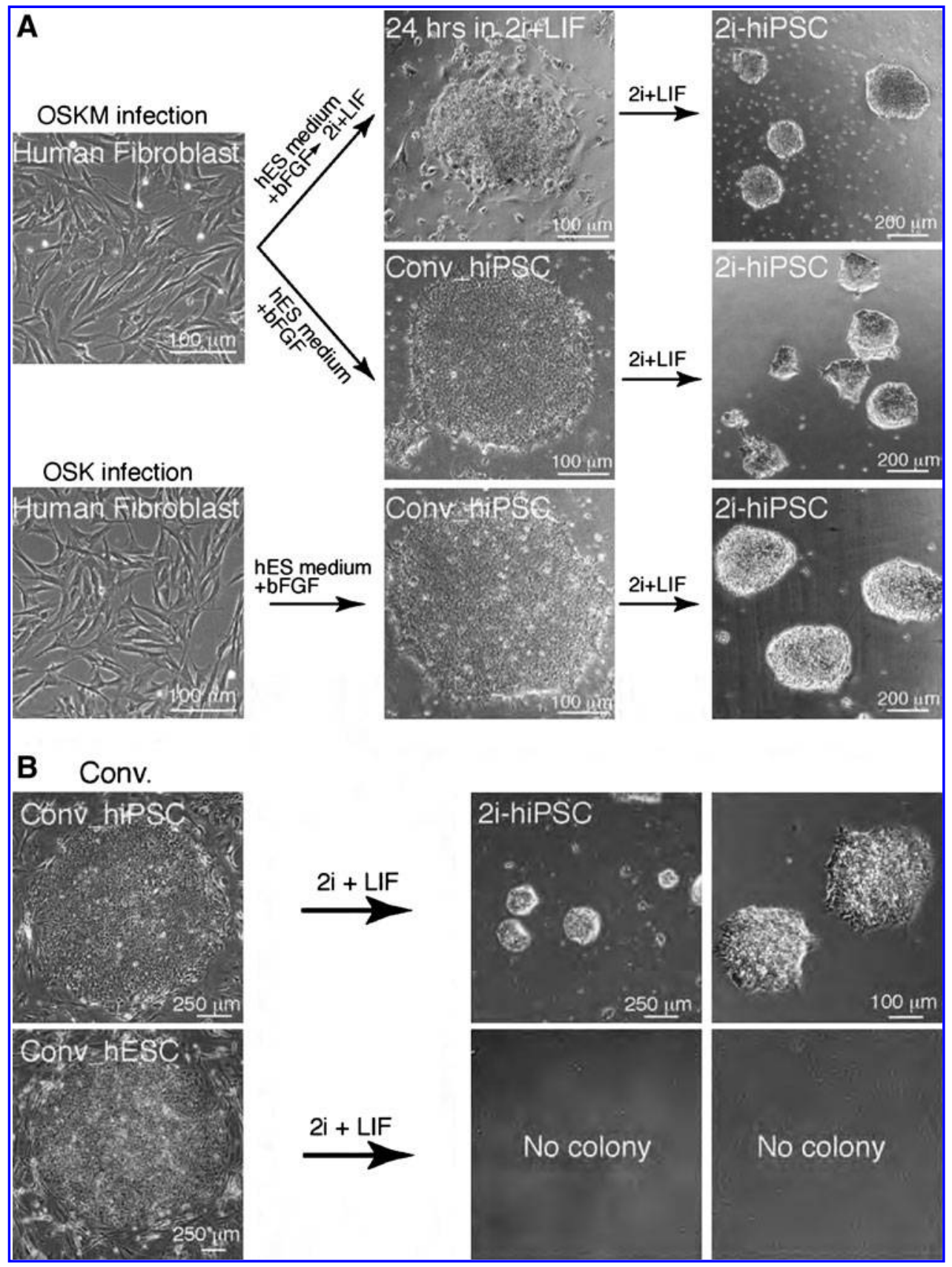

FIG. 2. Generation of human multipotent stem cell lines in $2 i+$ LIF medium. (A) Generation of human iPSCs (2i-hiPSCs) by reprogramming directly from primary fetal fibroblasts, conventional (Conv.) OSKM-hiPSCs, and conventional OSK-hiPSCs in $2 \mathrm{i}+\mathrm{LIF}$ medium (2i-hiPSCs). OSK(M): Oct4, Sox2, Klf4, cMyc. (B) 2i-hESCs cannot be generated from conventional hESCs in $2 i+$ LIF medium. hiPSC, human induced pluripotent stem cell; hESC, human embryonic stem cell. cells in $2 \mathrm{i}+\mathrm{LIF}$ medium is retained in conventional hiPSCs but not hESCs.

To address this, 3 independent OSKM Infr conventional iPSC lines derived from adult dermal cells HDF1388 [17] were cultured in $2 \mathrm{i}+\mathrm{LIF}$ medium. Importantly, 2i-iPSC lines were repeatedly isolated from each conventional cell line, even without exogenous OSKM integration. Therefore, it is likely that generation of 2i-hiPSCs but not 2i-hESCs reflects differential potential of cell reprogramming into multipotent stem cells in $2 i+L I F$ medium between conventional hiPSCs and hESCs.

\section{Gene expression in 2i-hiPSCs}

To explore the differential reprogramming potential of conventional hiPSCs and hESCs in $2 i+$ LIF medium, the global gene expression profiles of conventional hESCs and hiPSCs were compared with those of $2 i+$ hiPSCs by microarray analyses. Notably, 2i-hiPSCs exhibited significant differences from the conventional lines (Fig. 3A). 2i-hiPSCs showed reduced expression of pluripotent marker genes with the exception of SOX2. To verify the expression profile, selected genes were further investigated by RT-PCR (Fig. 3B). Pluripotent marker genes, OCT4, NANOG, NR0B1, and STELLA, were expressed at much reduced levels. However, consistent with the whole genome analysis, markedly higher levels of SOX2 expression were observed. Notably, the expression profiles and bowl-shaped colony morphology resembled those of PNSCs, which are capable of contribution into chimeras, and are defined by low level expression of the pluripotent marker genes, Oct4 and Nanog, and high level 
FIG. 3. Gene expression analysis of human iPSCs in $2 \mathrm{i}+$ LIF medium (2ihiPSCs). (A) Comparative analysis of global gene expression profile of conventional (Conv.) hESC, conventional hiPSC, and 2i-hiPSC lines by gene expression microarray assay. (B) Expression analysis of pluripotent marker genes in conventional hESC, conventional hiPSC, and 2i-hiPSC lines by reverse transcription (RT)-PCR. (C) Expression analysis of marker genes for primitive neural stem cells (PNSCs) in conventional hESC, conventional hiPSC, and 2i-hiPSC lines by RT-PCR. PCR, polymerase chain reaction.

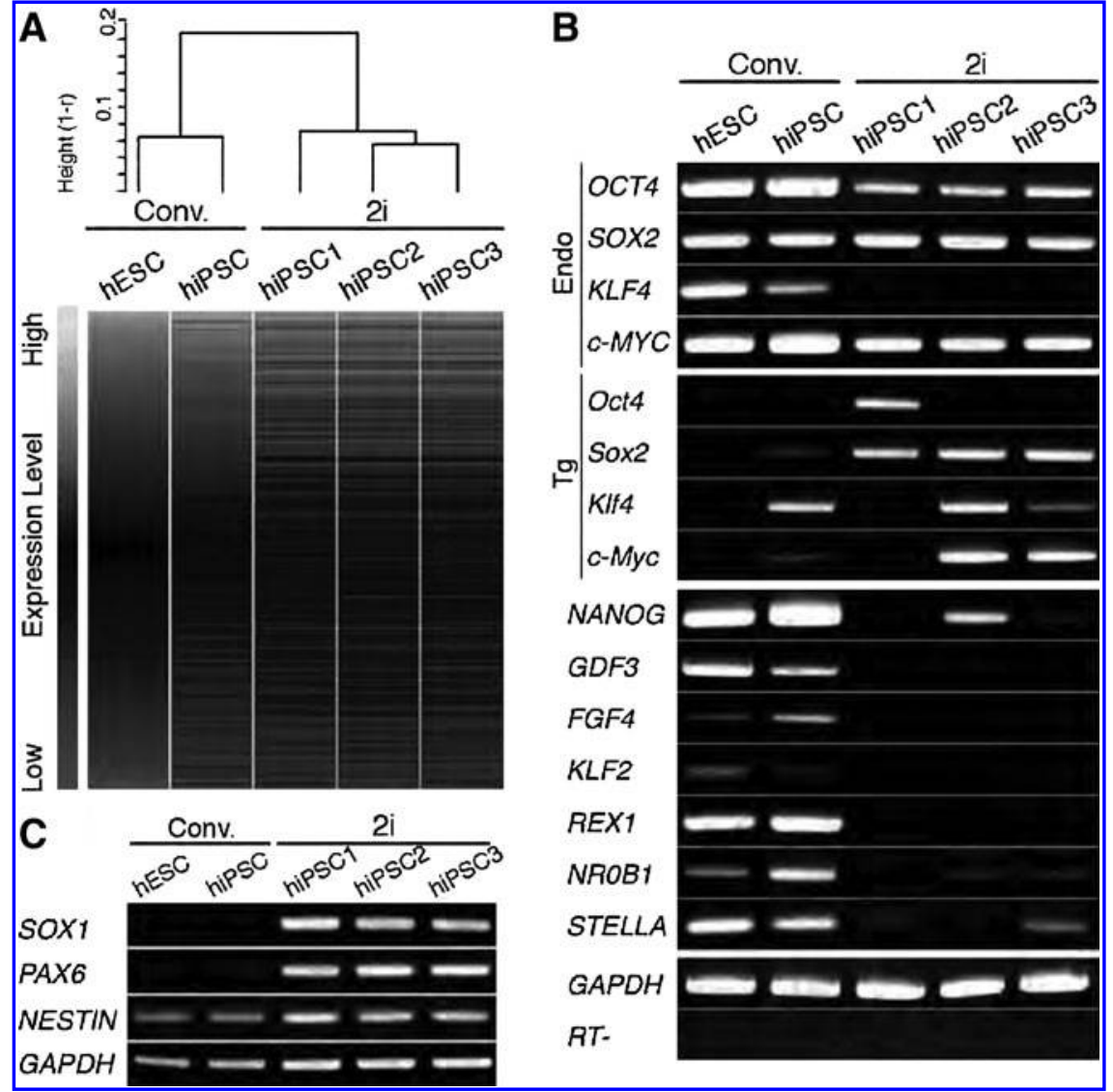

expression of the neuroectodermal marker genes, Nestin, Sox1, and Pax6 in mice [14]. Thus, expression of PNSC marker genes was analyzed. Notably, considerable highlevel SOX1 and PAX6 expression was observed in 2i-hiPSC lines but not conventional hESC and hiPSC lines (Fig. 3C). Expression of NESTIN was also detected in 2i-hiPSC lines, as well as in conventional hESC and hiPSC lines. Further, an inactivated $X$ chromosome was detected by H3K27me3 staining and RNA FISH analysis in each female 2i-hiPSC (Supplementary Fig. S1). Therefore, we conclude that 2ihiPSCs are phenotypically equivalent to mouse PNSCs, and not authentic mESCs. $2 i+$ LIF medium does not provide appropriate culture conditions for generating authentic hESCs, in contrast to generation of GS-mESCs.

\section{Emergence of differential lineages from hESC and hiPSC in $2 i+$ LIF medium}

Human PNSCs were generated from conventional hiPSCs but not hESCs. Conventional hESC lines, KhES1 and KhES3, were derived from Japanese male and female blastocysts; whereas conventional hiPSC lines and conventional Infr hiPSC lines were derived from fetal lung fibroblasts TIG1 and TIG3, and adult dermal fibroblasts HDF1388, respectively. It is, therefore, likely that origin of the somatic cells is not associated with differential emergence of differentiated cells from hESC and hiPSC lines in $2 \mathrm{i}+$ LIF medium. To explore how the differential response occurs, we examined expression of lineage marker genes, including PNSC markers, PAX6 and SOX1, in colonies picked for 2i-hiPSC gener- ation 5 days after culture in $2 \mathrm{i}+$ LIF medium (Fig. $4 \mathrm{~A}$ ). The reprogramming frequency to pick a colony was $\sim 30 \%$ (164 out of 536 colonies examined). Candidate colonies of $2 \mathrm{i}-$ hiPSCs that appeared during culture of conventional hiPSCs and Infr hiPSCs specifically expressed SOX1; whereas other cell populations expressed endodermal (GATA4), mesodermal (RUNX1 and T), and trophectodermal (CDX2) lineage markers associated with down-regulation of the pluripotent marker OCT4 (Fig. 4B). NESTIN was expressed in both picked and other colonies, whereas PAX6 was undetectable at the earliest conversion stage. Specific expression of the SOX1 protein in candidate $2 \mathrm{i}$-hiPSC colonies was verified by immunostaining (Fig. 4C). These findings in human cells were consistent with properties of PNSCs in the mouse. Sox1 is the earliest specific marker of neuroectoderm in mouse embryos in vivo [25]. Neural precursor cells were FACSpurified as Sox1-positive cell populations in differentiation of mouse Sox1-GFP knock-in ES cells in vitro [15]. These data support the notion that 2 i-hiPSCs are phenotypically equivalent to mouse PNSCs.

To examine which defined supplements in the culture medium were responsible for differential emergence of SOX1-positive colonies, conventional hiPSCs and hESCs were cultured in N2B27 medium with or without $2 i+$ LIF. Differential emergence of differentiated cells between hiPSCs and hESCs was detected only in N2B27 medium with $2 i+$ LIF (Supplementary Fig. S2). Withdrawal of $2 i+$ LIF induced cell differentiation into both neural and non-neural cells. Thus, we conclude that a combination of stimulatory effect of N2B27 on cell differentiation and chemical selection 


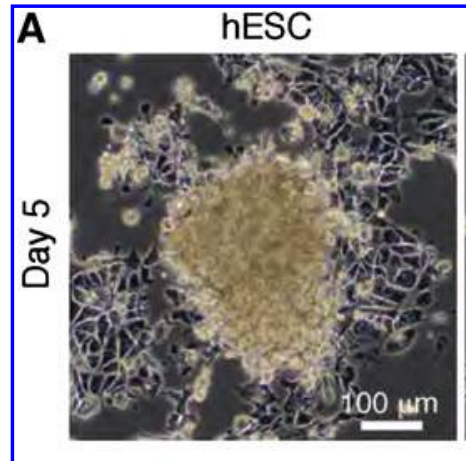

B

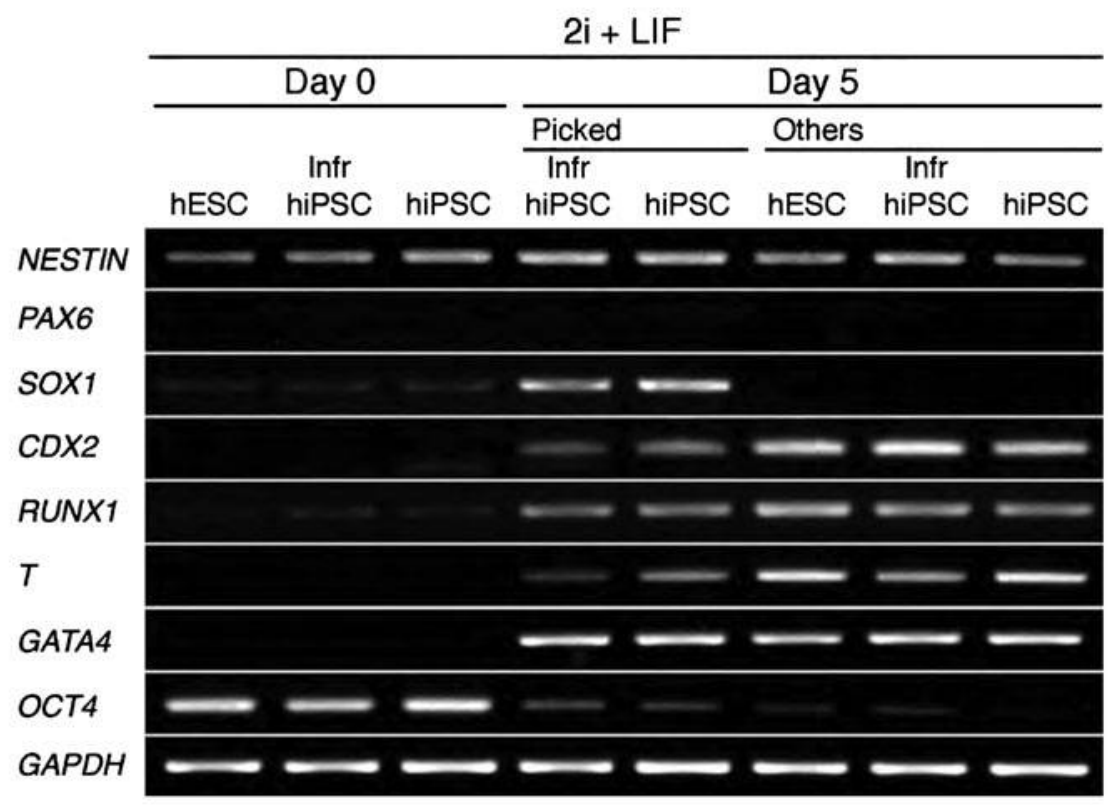

FIG. 4. Emergence of human iPSCs in $2 \mathrm{i}+$ LIF medium $(2 \mathrm{i}-$ hiPSCs). (A) Generation of $2 \mathrm{i}-$ hiPSCs in conventional hiPSC and integration-free (Infr) hiPSC but not conventional hESC after 5 days culture in $2 i+$ LIF medium. 2i-hiPSC colonies, donated by dotted lines (picked), were used for further RT-PCR analysis of 2i-hiPSC. (B) Comparative RT-PCR expression analysis of lineage marker genes in hESC, infr hiPSC, and hiPSC at day 0 and 5 after culture in $2 \mathrm{i}+$ LIF medium. NESTIN, PAX6, and SOX1 are marker genes for neuroectodermal tissues. (C) Expression of SOX1 protein in hiPSC colony after 5 days culture in $2 \mathrm{i}+\mathrm{LIF}$ medium. A 2i-hiPSC colony (picked) is demarked by dotted lines.

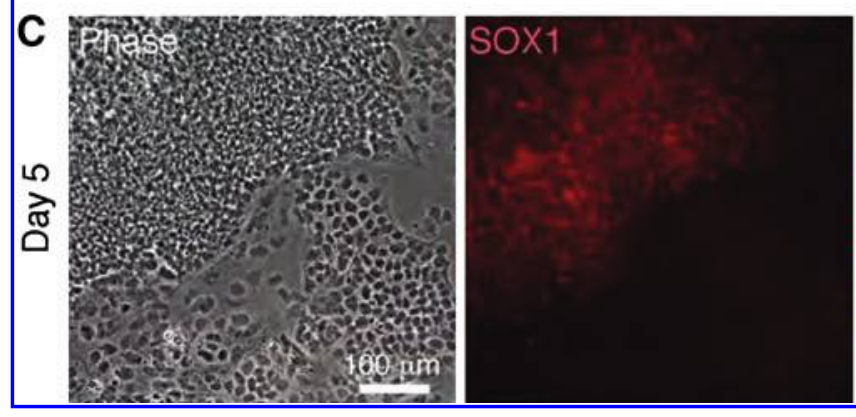

of $2 \mathrm{i}+$ LIF-resistant neural cells is required for $2 \mathrm{i}$-hiPSC generation.

\section{Characterization of multipotent 2i-hiPSCs}

To further characterize 2i-hiPSCs, pluripotent marker protein expression in undifferentiated human and mESCs was examined by immunostaining. 2i-hiPSCs were positive for OCT4, SOX2, and KLF4, weakly positive for NANOG, and negative for E-CADHERIN, SSEA1, SSEA4, and TRA-160 (Fig. 5A). Consistent with data on gene expression analyses (Fig. 3A, B), the protein expression profile of 2i-hiPSCs was clearly different from that of conventional hESCs which are positive for E-CADHERIN, SSEA4, and TRA-1-60. Next, to analyze the similarity of $2 \mathrm{i}$-hiPSCs to PNSCs, differentia-

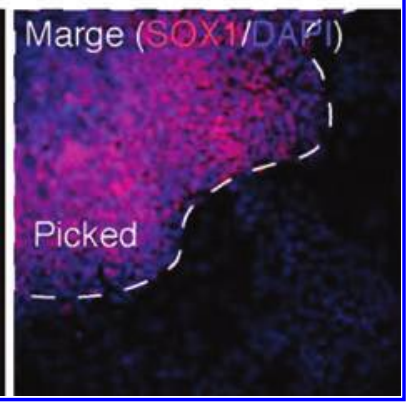

tion of 2i-hiPSCs was induced in vitro. Cystic embryoid bodies (EBs) were successfully generated within 5-7 day suspension culture for all 2i-hiPSC lines tested (Fig. 5B), thus indicating that $2 \mathrm{i}$-hiPSCs can differentiate at least into the endodermal lineage. Ectodermal differentiation was evidenced by efficient differentiation into neural cells, induced 4-5 days after withdrawal of chemicals, $2 i$ and LIF, from $2 i+$ LIF medium (Fig. 5B). Similar neural differentiation was induced several days after replacement of $2 i+$ LIF medium with DMEM-F12 medium containing FBS (data not shown).

To determine the type of neural cells, we performed immunostaining on the differentiated cells. TuJ1 and NF-Mpositive neuronal cells and GFAP-positive astrocytes were detected at a high frequency. O4-positive oligodendrocytes were also observed, albeit at a much reduced frequency (Fig. 


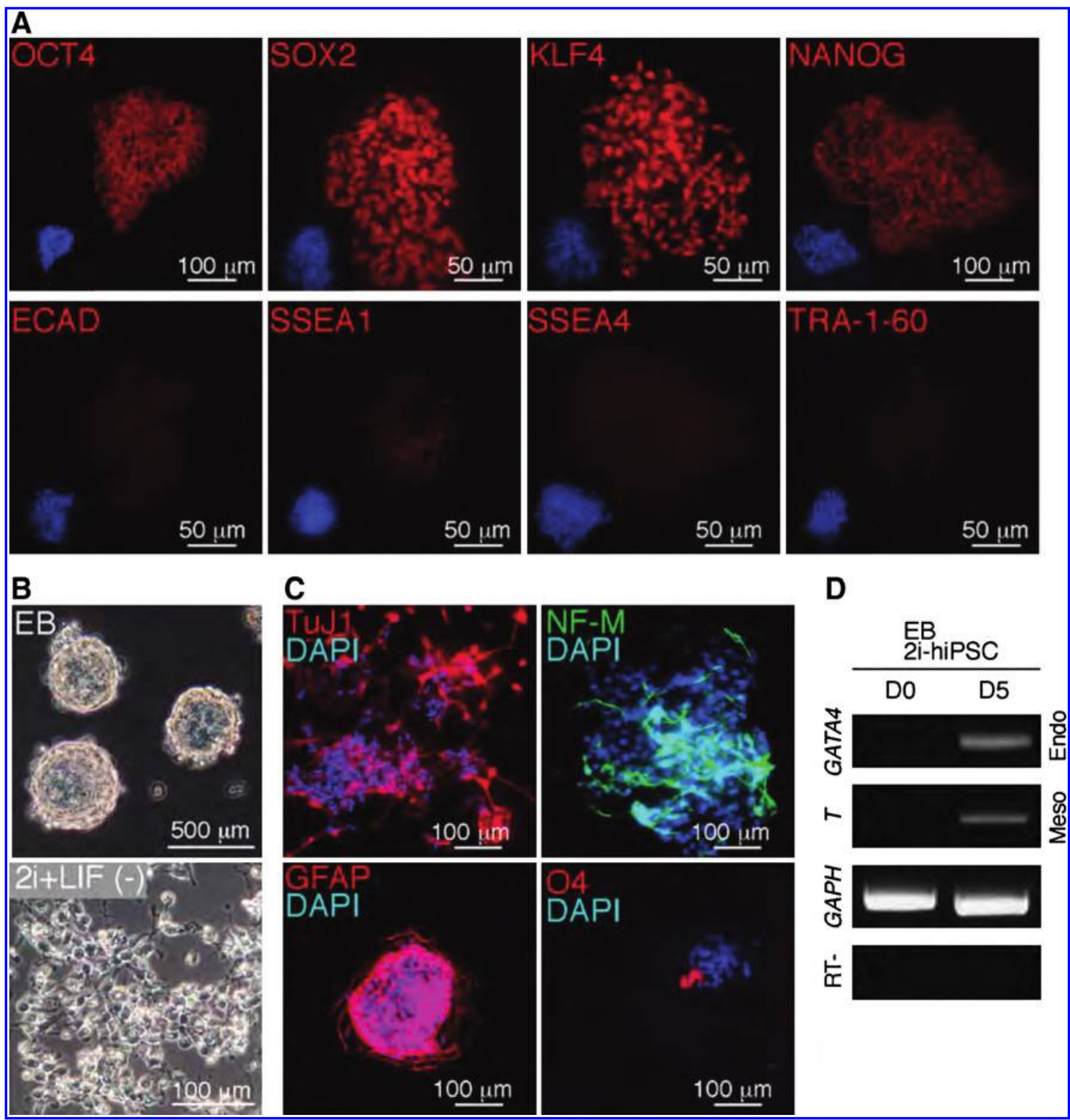

FIG. 5. Characterization of differentiated and undifferentiated 2i-hiPSCs in vitro. (A) Expression of pluripotent marker proteins (red) in 2i-hiPSCs detected by immunocytochemistry with specific antibodies. Cell nuclei are counterstained blue with DAPI. (B) Differentiation of 2i-hiPSCs by embryoid body (EB) formation in suspension culture, and into neural cells by culture in neurobasal N2B27 medium without $2 \mathrm{i}+$ LIF [2i+LIF (-)]. (C) Characterization of neural cell types by immunocytochemistry with specific antibodies. TuJ1 (neuron-specific class III beta-tubulin) and NF-M (neurofilament-M) are markers for neuron, GFAP (glial fibrillary acidic protein) is a marker for glia, and O4 is a marker for oligodendrocyte. Cell nuclei are counterstained blue with DAPI. (D) RT-PCR expression analysis of lineage marker genes in day 5 (D5) EB formed with 2ihiPSCs. GATA4 is a marker for endodermal derivatives, whereas $T$ is a marker for mesodermal derivatives. DAPI, 4,6diamidino-2-phenylindole.

5C). Thus, 2i-hiPSCs have the potential to differentiate into all types of neurons, astrocytes, and oligodendrocytes, as previously shown with mouse PNSCs [14]. Multi-lineage differentiation of 2i-hiPSCs was verified by expression of mesodermal $(T)$ and endodermal (GATA4) lineage marker genes in day 5 EBs (Fig. 5D). Experiments to demonstrate contribution of 2i-hiPSCs into normal embryonic development were not approved on ethical grounds, although efficient contribution of PNSCs into aggregation chimeras in mice suggests that $2 \mathrm{i}$-hiPSCs may well have the capacity to do so [26]. As an alternative approach, 2i-hiPSCs were implanted into kidney capsules of immunodeficient SCID mice to assess their in vivo teratoma forming ability. Notably, no teratomas was observed, from 10 independent implantations of undifferentiated and EB-formed 2i-hiPSCs, thus indicating that 2i-hiPSCs have considerably reduced potential for teratoma formation than their parental cells (Supplementary Fig. S3A), in contrast to teratoma formation with parental conventional hiPSCs (Supplementary Fig. S3B). Thus, we conclude that multi-potent $2 \mathrm{i}$-hiPSCs that are primitively 
committed to neural differentiation, similar to PNSCs in mice, may represent a suitable source of personalized replacement cells in practical regenerative medicine.

\section{Discussion}

We demonstrate here that a culture with $2 i+$ LIF medium induces reprogramming of flat-shaped human iPSCs into bowl-shaped PNSCs, which contrasts markedly to reprogramming of mESCs, iPSCs, and EpiSCs into mouse bowlshaped GS PSCs. Human iPSCs were different from miPSCs even with the same minimal extrinsic stimuli in $2 i+$ LIF medium (Fig. 6). In humans, 2i-hiPSCs resemble PNSCs in gene expression profile and multipotency. They can be stably expanded on matrigel-coated dishes without feeder cells by mechanical dissociation for $>50$ passages. Generation of $2 i-$ hiPSCs from conventional hiPSCs, but not the hESCs we used, reflects a tendency for hiPSCs toward neuroectodermal lineages. Multi-potential 2i-hiPSCs, capable of differentiating into neurons, astrocytes, and oligodendrocytes in vitro, did not form teratomas in vivo. Together, the results indicate that $2 \mathrm{i}$ hiPSCs are a promising source of cells for human neural research, pharmaceutical screening, and regenerative medicine.

\section{Difference between human and mouse PSCs in $2 i+$ LIF medium}

In rodents, $2 \mathrm{i}+$ LIF culture enriches GS PSCs characterized by uniform stem cell identity and germline competence $[1,3,4]$. This suggests that neuroectodermal differentiation induction by neurobasal N2B27 medium and differentiation inhibition by $2 \mathrm{i}$ and LIF are functionally balanced for tipping toward stemness defined by prolonged self-renewal in the undifferentiated state [1]. The same $2 i+$ LIF culture conditions induced a multi-potent PNSC state with conventional hiPSCs, differing substantially from the pluripotent ES state seen with conventional mEpiSCs (Fig. 1A) [21,22]. The differences could be explained by at least 2 possibilities: (1) hiPSCs and mEpiSCs contain different subpopulations of cells that preferentially respond to the $2 i+$ LIF culture condition, and (2) although hiPSCs and mEpiSCs exhibit similar flat-shaped colony morphology in a conventional state, hiPSCs retain a uniquely different molecular network sig- nature that serves to promote neural differentiation in $2 i+$ LIF medium.

In conventional and Infr iPSC populations, isolation of human PNSCs with $2 i+$ LIF medium demonstrates the possibility that subpopulations of cells directly reprogrammed into PNSCs are generated and stably maintained in human ES medium with bFGF, which is required for primary neural specification [15]. These PNSCs, directly reprogrammed from somatic cell by reinforced transient expression of OSK, are selected and expanded in $2 \mathrm{i}+\mathrm{LIF}$ medium. This could account for isolation of human PNSCs from hiPSCs but not hESCs.

In mouse PSCs, reprogramming of ESCs into PNSCs can be enhanced by inhibition of TGF $\beta$-related signaling mediated by substances including bone morphogenetic proteins (BMPs) as a default manner of neural fate specification [26]. Consistent with this, MEK inhibitor PD0325901, inhibiting neural differentiation through impeding ERK phosphorylation, functions to suppress neural commitment via the BMP pathway [1]. Therefore, another explanation is differential responses between mouse PSCs and hiPSCs to the extracellular signals introduced in $2 \mathrm{i}+\mathrm{LIF}$ medium. Commitment to neural differentiation occurs in fully reprogrammed pluripotent hiPSCs by imperfect inhibition even in the presence of PD0325901. If so, then commitment of PSCs to form neural cells may be negatively regulated by not only the TGF $\beta$-related signaling pathway but also other signaling pathways in the humans, as demonstrated by differential fate determination in mEpiSCs and hESCs [27] and neural conversion of hESCs and hiPSCs using the dual-SMADinhibition protocol [28]. Recently, it was reported that hESCs were rapidly converted to homogenous PNSCs by a synergistic effect of inhibitors to GSK3, TGF $\beta$, and Notch signaling pathways [29]. hESC (H1, HUES9, and HUES1)-derived PNSC lines efficiently ( $>80 \%$ ) generated by 1 week culture with N2B27 supplemented with CHIR99021, SB431542, and LIF were characterized by intensive analyses of expression of rosette-type neural stem cell (NSC), central nerve system NSC, anterior neural, and midbrain marker proteins by immunostaining, and midbrain marker genes by RT-PCR, and functional assays in vitro and in vivo. 2i-hiPSCs resemble hESC-derived PNSCs [29] in the expression profile of marker genes and proteins (Supplementary Fig. S4). Differential

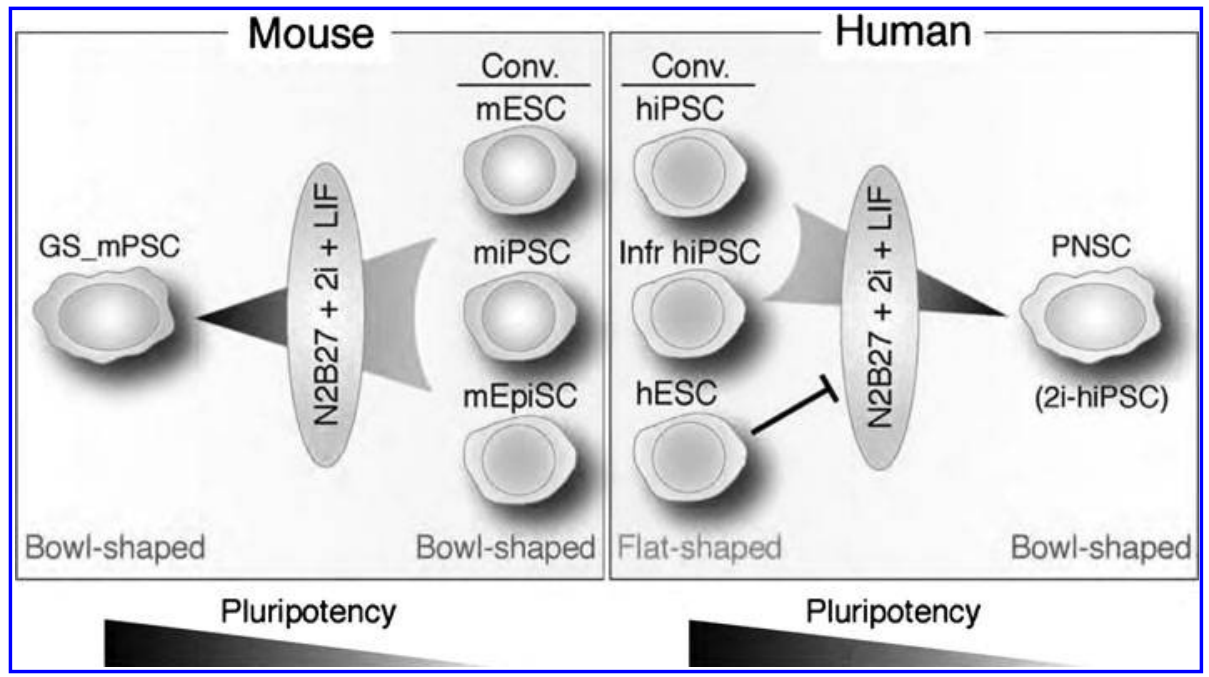

FIG. 6. Schema of differential emergence of PSCs in $2 i+$ LIF medium. In humans, PNSC emerge from conventional (Conv.) and Infr hiPSC, but not hESC, whereas mouse GS PSCs are generated from mESC, miPSC, and mEpiSC. 
requirements between human and mESCs for differentiation into PNSCs may reflect a distinct physiology between mouse and hESCs.

\section{Difference between human iPSCs and ESCs in $2 i+$ LIF medium}

Human iPSCs are functionally and molecularly indistinguishable from hESCs on the basis of multipotency, global gene expression, and DNA methylation profile $[5,6,30]$. Recent intensive research, however, demonstrated differential gene expression and DNA methylation between ESCs and iPSCs. Early passage iPSCs retained epigenetic memory inherited from their somatic cell origin, and dominantly differentiated into the cell type of origin. However, somatic epigenetic memory disappeared on continuous sub-culturing for both the human [31] and mouse cells [32]. Further analyses of whole-genome profiles of DNA methylation revealed that substantial hyper- and hypo-methylated $\mathrm{CpG}$ islands (differentially methylated regions) generated by aberrant and incomplete reprogramming existed in hiPSCs compared with hESCs [33,34]. Therefore, differential differentiation potentials between hiPSCs and hESCs in $2 i+$ LIF medium may reflect differential epigenetic memory generated through inadequate reprogramming of somatic cells into hiPSCs. Here, we have ruled out the possibility of residual somatic epigenetic memory by using late-passage hiPSCs derived from 2 different somatic cell types. It is, however, possible that the hiPSCs cell lines we used are primed for differentiation into neuroectodermal lineages by DNA hypo-methylation of the promoter regions of key genes for neural differentiation. This, however, is not consistent with previous reports of low differentiation efficiency of hiPSCs into neural cells [35]. If so, then 2i-hESC lines may be established from some hESC lines, which have robust potentials of differentiating into neural cells [34] in $2 i+$ LIF medium.

\section{Stable maintenance of multipotent 2i-hiPSC lines}

An intriguing property of 2i-hiPSCs is lack of expression of SSEA4 and E-CADHERIN, which is different from conventional hESCs and hiPSCs (Fig. 5A). In conventional hESCs, $\mathrm{Ca}^{2+}$-dependent E-CADHERIN-mediated cell-to-cell adhesion prevents apoptosis by ROCK/myosin hyperactivation [36]. No improvement in survival and proliferation of 2i-hiPSCs was detected with the treatment of ROCK inhibitor Y27632, or Myosin IIA inhibitor Blebbistatin [23,24] through subculture by enzymatic dissociation (data not shown). This is consistent with our observation that 2i-hiPSCs can be stably maintained by mechanical dissociation into $40-50$ cell clusters but not enzymatic dissociation into single cells. However, it is consistent with no expression of ECADHERIN in 2i-hiPSCs. Thus, it is speculated that other cell-to-cell adhesion molecules involved in inhibition of apoptosis, such as N-CADHERIN [29,37], may play an important role in survival of $2 \mathrm{i}$-hiPSCs and human PNSCs in $2 \mathrm{i}+\mathrm{LIF}$ medium. The putative role of cell adhesion molecule function, in maintaining a multipotent state of 2i-hiPSCs, is supported by induction of neural differentiation in chemically defined serum-free, feeder-layer free, and low-density culture conditions [26] and in enzymatic dissociation into single cells in $2 i+$ LIF medium (data not shown).

\section{Establishment of authentic human iPSCs from conventional hiPSCs}

A few studies demonstrated that naïve hESCs or hiPSCs, which are an authentic counterpart of mESCs, could be generated but not maintained during long-term in vitro culture $[12,13]$. Naïve hESCs can be defined by bowl-shaped colony morphology, 2 active $X$ chromosomes in female cells, pluripotency, and gene expression and epigenetic profile closely similar to those of mESCs. With regard to $\mathrm{X}$-chromosome state, 2 active $\mathrm{X}$ chromosomes in an XX female cell are a hallmark of naïve ESCs in the mouse. In humans, one of $2 X$ chromosomes is inactivated in conventional hESCs [20,38]. Interestingly, reducing the oxygen concentration from conventional $20 \%$ to physiological $5 \%$ in culture induces derivation of hESCs retaining 2 active $X$ chromosomes [39]. In miPSCs, it is evident that an adequate culture condition enables establishment of naïve iPSC lines from various types of somatic cells by forced expression of transcription factors, OSKM or OSK, thus suggesting that transient expression of the transcription factors is sufficient for generating naïve hiPSCs with 2 active $X$ chromosomes in females. This suggests that naïve hiPSC could be established if appropriate specialized culture conditions were found and applied, that differ from current hESC culture conditions with KSR and bFGF. Establishment of naïve hiPSCs would provide a significant contribution to the study of mechanisms involved in early human embryonic development and regenerative medicine.

\section{Acknowledgments}

The authors thank Dr. Gen Kondoh and Ms. Hitomi Watanabe for generating chimeras, Dr. Kathrin Plath for the probe DNA to human XIST, and Dr. Takashi Sado for XIST RNA FISH. This work was partly funded by grants from the Japan Society for the Promotion of Science, the Ministry of Education, Culture, Sports, Science, and Technology and the Core Research for Evolutional Science and Technology (Japan Science and Technology Agency) to T.T. K.H is a fellow of JSPS.

\section{Author Disclosure Statement}

The authors declare that they have no conflict of interest.

\section{References}

1. Ying QL, J Wray, J Nichols, L Batlle-Morera, B Doble, J Woodgett, P Cohen and A Smith. (2008). The ground state of embryonic stem cell self-renewal. Nature 453:519-523.

2. Nichols J, K Jones, JM Phillips, SA Newland, M Roode, W Mansfield, A Smith and A Cooke. (2009). Validated germline-competent embryonic stem cell lines from nonobese diabetic mice. Nat Med 15:814-818.

3. Buehr M, S Meek, K Blair, J Yang, J Ure, J Silva, R McLay, J Hall, QL Ying and A Smith. (2008). Capture of authentic embryonic stem cells from rat blastocysts. Cell 135:12871298.

4. Li P, C Tong, R Mehrian-Shai, L Jia, N Wu, Y Yan, RE Maxson, EN Schulze, H Song, et al. (2008). Germline competent embryonic stem cells derived from rat blastocysts. Cell 135:1299-1310. 
5. Takahashi K, K Tanabe, M Ohnuki, M Narita, T Ichisaka, K Tomoda and S Yamanaka. (2007). Induction of pluripotent stem cells from adult human fibroblasts by defined factors. Cell 131:861-872.

6. Lowry WE, L Richter, R Yachechko, AD Pyle, J Tchieu, R Sridharan, AT Clark and K Plath. (2008). Generation of human induced pluripotent stem cells from dermal fibroblasts. Proc Natl Acad Sci USA 105:2883-2888.

7. Takahashi K and S Yamanaka. (2006). Induction of pluripotent stem cells from mouse embryonic and adult fibroblast cultures by defined factors. Cell 126:663-676.

8. Brons IG, LE Smithers, ME Trotter, P Rugg-Gunn, B Sun, SM Chuva de Sousa Lopes, SK Howlett, A Clarkson, L AhrlundRichter, RA Pedersen and L Vallier. (2007). Derivation of pluripotent epiblast stem cells from mammalian embryos. Nature 448:191-195.

9. Tesar PJ, JG Chenoweth, FA Brook, TJ Davies, EP Evans, DL Mack, RL Gardner and RD McKay. (2007). New cell lines from mouse epiblast share defining features with human embryonic stem cells. Nature 448:196-199.

10. Niwa H. (2007). How is pluripotency determined and maintained? Development 134:635-646.

11. Silva J and A Smith. (2008). Capturing pluripotency. Cell 132:532-536.

12. Hanna J, AW Cheng, K Saha, J Kim, CJ Lengner, F Soldner, JP Cassady, J Muffat, BW Carey and R Jaenisch. (2010). Human embryonic stem cells with biological and epigenetic characteristics similar to those of mouse ESCs. Proc Natl Acad Sci USA 107:9222-9227.

13. Xu Y, X Zhu, HS Hahm, W Wei, E Hao, A Hayek and S Ding. (2010). Revealing a core signaling regulatory mechanism for pluripotent stem cell survival and self-renewal by small molecules. Proc Natl Acad Sci USA 107:8129-8134.

14. Conti L and E Cattaneo. (2010). Neural stem cell systems: physiological players or in vitro entities? Nat Rev Neurosci 11:176-187.

15. Ying QL, M Stavridis, D Griffiths, M Li and A Smith. (2003). Conversion of embryonic stem cells into neuroectodermal precursors in adherent monoculture. Nat Biotechnol 21:183186.

16. Nagata S, M Toyoda, S Yamaguchi, K Hirano, H Makino, K Nishino, Y Miyagawa, H Okita, N Kiyokawa, et al. (2009). Efficient reprogramming of human and mouse primary extra-embryonic cells to pluripotent stem cells. Genes Cells 14:1395-1404.

17. Okita K, Y Matsumura, Y Sato, A Okada, A Morizane, S Okamoto, H Hong, M Nakagawa, K Tanabe, et al. (2011). A simple method to generate integration-free human iPA cells. Nat Methods 8:409-412.

18. Suemori H, K Yasuchika, K Hasegawa, T Fujioka, N Tsuneyoshi and N Nakatsuji. (2006). Efficient establishment of human embryonic stem cell lines and long-term maintenance with stable karyotype by enzymatic bulk passage. Biochem Biophys Res Commun 345:926-932.

19. Tada M, T Tada, L Lefebvre, SC Barton and MA Surani. (1997). Embryonic germ cells induce epigenetic reprogramming of somatic nucleus in hybrid cells. EMBO J 16:65106520 .

20. Tchieu J, E Kuoy, MH Chin, H Trinh, M Patterson, SP Sherman, O Aimiuwu, A Lindgren, S Hakimian, et al. (2010). Female human iPSCs retain an inactive $X$ chromosome. Cell Stem Cell 7:329-342.

21. Guo G, J Yang, J Nichols, JS Hall, I Eyres, W Mansfield and A Smith (2009). Klf4 reverts developmentally programmed restriction of ground state pluripotency. Development 136:1063-1069.

22. Han DW, N Tapia, JY Joo, B Greber, MJ Arauzo-Bravo, C Bernemann, K Ko, G Wu, M Stehling, JT Do and HR Scholer. (2010). Epiblast stem cell subpopulations represent mouse embryos of distinct pregastrulation stages. Cell 143:617-627.

23. Watanabe K, M Ueno, D Kamiya, A Nishiyama, M Matsumura, T Wataya, JB Takahashi, S Nishikawa, K Muguruma and Y Sasai. (2007). A ROCK inhibitor permits survival of dissociated human embryonic stem cells. Nat Biotechnol 25:681-686.

24. Li D, J Zhou, L Wang, ME Shin, P Su, X Lei, H Kuang, W Guo, H Yang, et al. (2010). Integrated biochemical and mechanical signals regulate multifaceted human embryonic stem cell functions. J Cell Biol 191:631-644.

25. Wood HB and V Episkopou. (1999). Comparative expression of the mouse Sox1, Sox2 and Sox3 genes from pre-gastrulation to early somite stages. Mech Dev 86:197-201.

26. Tropepe V, S Hitoshi, C Sirard, TW Mak, J Rossant and D van der Kooy. (2001). Direct neural fate specification from embryonic stem cells: a primitive mammalian neural stem cell stage acquired through a default mechanism. Neuron 30:65-78.

27. Greber B, G Wu, C Bernemann, JY Joo, DW Han, K Ko, N Tapia, D Sabour, J Sterneckert, P Tesar and HR Scholer. (2010). Conserved and divergent roles of FGF signaling in mouse epiblast stem cells and human embryonic stem cells. Cell Stem Cell 6:215-226.

28. Chambers SM, CA Fasano, EP Papapetrou, M Tomishima, M Sadelain and L Studer. (2009). Highly efficient neural conversion of human ES and iPS cells by dual inhibition of SMAD signaling. Nat Biotechnol 27:275-280.

29. Li W, W Sun, Y Zhang, W Wei, R Ambasudhan, P Xia, M Talantova, T Lin, J Kim, et al. (2011). Rapid induction and long-term self-renewal of primitive neural precursors from human embryonic stem cells by small molecule inhibitors. Proc Natl Acad Sci USA 108:8299-8304.

30. Bock C, E Kiskinis, G Verstappen, H Gu, G Boulting, ZD Smith, M Ziller, GF Croft, MW Amoroso, et al. (2011). Reference maps of human ES and iPS cell variation enable high-throughput characterization of pluripotent cell lines. Cell 144:439-452.

31. Chin MH, MJ Mason, W Xie, S Volinia, M Singer, C Peterson, G Ambartsumyan, O Aimiuwu, L Richter, et al. (2009). Induced pluripotent stem cells and embryonic stem cells are distinguished by gene expression signatures. Cell Stem Cell 5:111-123.

32. Polo JM, S Liu, ME Figueroa, W Kulalert, S Eminli, KY Tan, E Apostolou, M Stadtfeld, Y Li, et al. (2010). Cell type of origin influences the molecular and functional properties of mouse induced pluripotent stem cells. Nat Biotechnol 28: 848-855.

33. Doi A, IH Park, B Wen, P Murakami, MJ Aryee, R Irizarry, B Herb, C Ladd-Acosta, J Rho, et al. (2009). Differential methylation of tissue- and cancer-specific CpG island shores distinguishes human induced pluripotent stem cells, embryonic stem cells and fibroblasts. Nat Genet 41:13501353.

34. Lister R, M Pelizzola, YS Kida, RD Hawkins, JR Nery, G Hon, J Antosiewicz-Bourget, R O'Malley, R Castanon, et al. (2011). Hotspots of aberrant epigenomic reprogramming in human induced pluripotent stem cells. Nature 471:68-73. 
35. Hu BY, JP Weick, J Yu, LX Ma, XQ Zhang, JA Thomson and SC Zhang. (2010). Neural differentiation of human induced pluripotent stem cells follows developmental principles but with variable potency. Proc Natl Acad Sci USA 107:43354340.

36. Ohgushi $\mathrm{M}, \mathrm{M}$ Matsumura, $\mathrm{M}$ Eiraku, K Murakami, $\mathrm{T}$ Aramaki, A Nishiyama, K Muguruma, T Nakano, H Suga, et al. (2010). Molecular pathway and cell state responsible for dissociation-induced apoptosis in human pluripotent stem cells. Cell Stem Cell 7:225-239.

37. Elkabetz Y, G Panagiotakos, G Al Shamy, ND Socci, V Tabar and L Studer. (2008). Human ES cell-derived neural rosettes reveal a functionally distinct early neural stem cell stage. Genes Dev 22:152-165.

38. Silva SS, RK Rowntree, S Mekhoubad and JT Lee. (2008). Xchromosome inactivation and epigenetic fluidity in human embryonic stem cells. Proc Natl Acad Sci USA 105:4820-4825.

39. Lengner CJ, AA Gimelbrant, JA Erwin, AW Cheng, MG Guenther, GG Welstead, R Alagappan, GM Frampton, P Xu, et al. (2010). Derivation of pre-X inactivation human em- bryonic stem cells under physiological oxygen concentrations. Cell 141:872-883.

Address correspondence to: Dr. Takashi Tada Laboratory of Stem Cell Engineering Institute for Frontier Medical Sciences

Kyoto University

53 Kawahara-cho

Shogo-in

Sakyo-ku

Kyoto 606-8507

Japan

E-mail: ttada@frontier.kyoto-u.ac.jp

Received for publication June 7, 2011

Accepted after revision August 27, 2011

Prepublished on Liebert Instant Online September 1, 2011 
This article has been cited by:

1. Alejandro De Los Angeles, Yuin-Han Loh, Paul J Tesar, George Q Daley. 2012. Accessing naïve human pluripotency. Current Opinion in Genetics \& Development. [CrossRef] 